Aus der Medizinischen Klinik der Universität in Straßburg i. E. (z. Z. Direktor: Prof. Dr. Krehl.)

\section{Weitere Beobachtungen über Untersuchung des Bluts auf Typhusbazillen und auf Agglutination.}

Von cand. med. Wolfgang Veil.

Die Diagnostik des Typhus scheint erledigt zu sein, weil in der Tat der letzte Schlüssel gefunden wurde, mit dessen Hilfe man zu einer sicheren Erkennung der Krankheit gelangen kann, nämlich die Züchtung der Bazillen aus dem Blute. Und doch müssen wir gerade jetzt nach Entdeckung und Einfülurung der neuen bakteriologischen Methoden mit der Prüfung unserer diagnostischen Maßnahmen von vorn anfangen. Denn es gilt auf der einen Seite die praktische, d. h. in der allgemeinen ärztlichen Tätigkeit durchführbare Verwendbarkeit der neuen Verfahren, auf der andern ihr Verhältnis zu den altbewährten Methoden zu studieren. Von dem einzelnen Krankheitssymptom muß festgestellt werden, wie weit es prozentarisch in jeder Periode einer Krankheit zu ihrer Erkennung beiträgt. Danach ist sein Wert für die Diagnose einzuschätzen. Denn deren Sicherheit beruht auf der Bedeutung jeder einzelnen der vorhandenen Krankheitserscheinungen, sowie auf ihrer Vereinigung. Das Ziel unserer Arbeit ist, daß die Diagnose zu einer Berechnung aus bestimmten Größen wird.

Durch die Hilfe der Straßburger Untersuchungsstation der südwestdeutschen Typhusbekämpfung ist die Klinik in die günstige Lage versetzt, umfangreiche Untersuchungen von erfahrenen Fachleuten ausgeführt zu erhalten. Brion und Kayser ${ }^{1}$ ) haben 1905 über die bis April des vergangenen Jahres beobachteten Fälle berichtet. Im folgenden sind die Befunde bei 210 Kranken geschildert, die seitdem beobachtet wurden.

Die Technik der Blutuntersuchung ist von Kayser") im letzten Jahrgang der Mtinchener medizinischen Wochenschrift beschrieben. Etwa 2-2,5 ccm Blut, das zweckmäbigst durch Venenpunktion aus der Armvene gewonnen wird, wird mit $5 \mathrm{ccm}$ reiner, normaler Rindergalle vermischt, wie sie die "Typhus-Galleröhre KayserGonradi“ enthält. Nachdem die Röhre 14-20 Stunden bei etwa $37^{\circ} \mathrm{C}$ gestanden hat, kann die Verarbeitung nach einer der bekannten Züchtungsmethoden erfolgen. (Ausstriche auf die Endosche Fuchsin-Milchzucker-Agarplatte oder den Drigalski-Con radischen Lackmusboden oder dgl.). Hauptbedingung für das Gelingen der Züchtung ist der AusschluB von bakteriellen Verunreinigungen bei der Anwendung des Verfahrens.

Von den untersuchten 210 Kranken, bei denen nach der klinischen Beobachtung und der ganzen Art des Krankheitsverlaufs mit Sicherheit Abdominaltyphus angenommen werden mubte, haben nur $4=2 \%$ bis zu ihrer Entlassung aus dem Krankenhaus sowohl Typhusbazillen im Blut als auch Agglutination völlig vermissen lassen. Bei einem dieser Kranken wurde die klinische Diagnose dadurch noch besonders gestützt, da0 Paratyphusbazillen $B$ aus dem Stuhle gezüchtet werden konnten. Bakterizidieversuche wurden leider bei keinem der vier Kranken angestellt. Zwei weitere Fälle schließen sich den eben angeführten an, indem sie nur vorübergehend eine schwache Agglutinationsreaktion aufwiesen, Bazillenbefund im Blute hingegen nie gewährten und von bakteriologischen Fachleuten nach diesen Befunden nicht als Typhuskranke angesehen werden konnten. Klinisch war die Diagnose Abdominaltyphus einwandfrei zu stellen und fand bei dem einen der Fälle später doch noch eine bakteriologische Bestätigung dadurch, daß der Pfeiffersche Bakterizidieversuch positiv ausfiel $(0,01$ Patientenserum schützte ein Meerschweinchen vor der vierfach letalen Dosis). Bei dem andern der Patienten klinisch dem ausgesprochensten "Nephrotyphus“ mit Roseolen, Milztumor, relativer Pulsverlangsamung, Indicanurie

1) Brion und Kayser, Deutsches Archuv für klinische Medizin Bd 85 . 2) Kayser. Manchener medizinische Wochenschrift 1906, No. 17 und 40 , sowie MajJunl 1907 . und starker, akuter, hämorrhagischer Nephritis - fiel der ebenfalls angestellte Bakterizidieversuch negativ aus.

Um völlig eindeutige Verhältnisse zu haben, trennten wir für unsere Betrachtung die durch Typhus- und die durch Paratyphusbazillen hervorgerufenen Erkrankungen. Auf die letzteren soll zum Schlub noch eingegangen werden. Die Infektionen mit Paratyphusbazillen $A$ und $B$ traten an Häufig. keit bedeutend zurück; auf etwa 200 Typhusfälle sahen wir zwölf Erkrankungen an Paratyphus.

Für die folgenden Untersuchungen haben wir unsere Fälle nach ihrem Verlauf in leichte, mittelschwere und schwere eingeteilt. Großen Wert mußten wir auf die Berücksichtigung der zeitlichen Verhältnisse legen. Ein Vergleich der anamnestischen Notizen der Klinik und des Instituts für südwestdeutsche Typhusbekämpfung, die uns Herr Dr. Kayser in zuvorkom. mendster Weise zur Verfügung stellte, zeigte aufs neue die Schwierigkeit, den Beginn der Erkrankung festzulegen. Unter Zugrundlegen der Erfahrung, daß im allgemeinen die bei Sektionsbefunden festgelegten Darmveränderungen auf einen früheren Invasionstermin hinwiesen als die anamnestischen Daten [vgl. die Arbeit v. Wennagel ${ }^{1}$ ]. habe ich jedesmal die frühest angesetzten Termine für den Krankheitsbeginn angenommen, sei es, daß diese den Aufzeichnungen der Klinik, sei es, daß sie denen des Hygienischen Instituts entstammten.

Die Gesichtspunkte, unter denen wir die Fälle nach ihrer Verlaufsart unterschieden, eine Einteilung, die zweifellos immer unter einer gewissen Willkür leidet, waren folgende: Unter die leichten Fälle wurden einmal die abortiv verlaufenden, ferner diejenigen gerechnet, bei denen die Temperatur $38^{\circ}$ nur wenig überschritt und das ganze Krankheitsbild sehr leicht war. Als schwer, resp. sehr schwer wurden diejenigen Fälle bezeichnet, bei denen die Temperatur abnorm hoch war und von vornherein die Krankheit einen bedrohlicheren Verlauf nahm, teils infolge stark ausgeprägter typhöser Symptome, teils infolge von erheblicheren. Kreislaufstörungen. Alle anderen Fälle galten als mittelschwer. Bei dieser Einteilung spielt der Ausgang der Krankheit, sei es, daß er sich ad restitutionem, sei es ad exitum letalem wandte, keine besondere Rolle. Die meisten Todesfälle befinden sich naturgemäß unter den schweren Fällen, doch sind auch unter den mittelschweren Fällen solche, die mit dem Tod endeten infolge von Perforationsperitonitis, Enterorrhagien und Embolien.

Tabelle I. Typhus abdominalis.

Uebersicht uber die Ergebnisse der Blutzüchtung und der Agglutination in der ersten Krankheitswoche.

\begin{tabular}{|c|c|c|c|c|c|}
\hline \multirow{2}{*}{$\begin{array}{l}\text { Verlaufsart } \\
\text { der Fälle }\end{array}$} & \multirow{2}{*}{$\begin{array}{c}\text { Zahl der } \\
\text { untersuch- } \\
\text { ten Falle }\end{array}$} & \multicolumn{2}{|c|}{ Zächtung } & \multicolumn{2}{|c|}{ Agglutination } \\
\hline & & $\begin{array}{l}\text { vorgenommen } \\
\text { in }\end{array}$ & $\underset{\text { In }}{\text { gelungen }}$ & $\begin{array}{l}\text { vorgenommen } \\
\text { in }\end{array}$ & $\begin{array}{c}\text { gelungen } \\
\text { in }\end{array}$ \\
\hline $\begin{array}{c}\text { Jeichte } \\
\text { mittelschwere } \\
\text { schwere }\end{array}$ & $\begin{array}{r}4 \\
24 \\
8 \\
\end{array}$ & $\begin{array}{r}4 \\
21 \\
7 \\
\end{array}$ & $\begin{aligned} 2 & =50 \% \\
16 & =76 \% \\
7 & =100 \%\end{aligned}$ & $\begin{array}{r}4 \\
24 \\
8 \\
\end{array}$ & $\begin{aligned} 3 & =75 \% \\
12 & =50 \% \\
4 & =50 \%\end{aligned}$ \\
\hline Summa & 36 & 32 & $25=78 \%$ & 36 & $18=50 \%$ \\
\hline
\end{tabular}

Man sieht also von neuem bestätigt, was Brion und Kayser schon hervorgehoben hatten, daß für die Frühdiagnose des Typhus die Blutzüchtung völlig die Situa tion beherrscht, während ein negativer Ausfall der Agglutinationsreaktion nur sehr bedingt $z u$ verwenden is t. Recht interessant ist das verschiedene, gewissermaßen gegensätzliche Verhalten der schweren und leichten Fälle. Die Blutzüchtung hat um so gröbere Chancen, ein positives Ergebnis zu zeigen, je schwerer der Krankheitszustand des Untersuchten ist. Würde man nur diejenigen Fälle heranziehen, die sich schon für die ärztliche Betrachtung als sehr ernste erweisen, so versagte die Blutzüchtung nie. Anderseits fällt die Agglutinationsreaktion um so wahrscheinlicher in der ersten Woche negativ aus, je sicherer die Blutzüchtung positiv wird, d. h. je schwerer die Infektion sich darstellt.

Es liegt dabei natïrlich die Vorstellung sehr nahe, daß die leichten Fälle eben doch deswegen leicht verlaufen, weil in dem erkrankten Körper irgendwelche der Bildung der agglutinierenden Substanzen parallel verlaufende Vorgänge sich abspielen, welche der Tätigkeit der Mikroorganismen Schranken

1) Wen nage1, Archıv fär klinische Medizın Bd. 87. 
setzen. Etwas Genaueres dürfte sich bei unserer völligen Unkenntnis von den Vorgängen der Heilung nicht sagen lassen.

Tabelle II. Typhus abdominalis.

Uebersicht über die Ergebnisse der Blutzüchtung und der Agglutination in der zweiten Krankheitswoche.

\begin{tabular}{|c|c|c|c|c|c|}
\hline \multirow{2}{*}{$\begin{array}{l}\text { Verlaufsart } \\
\text { der Fälle }\end{array}$} & \multirow{2}{*}{$\begin{array}{c}\text { Zahl der } \\
\text { untersuch. } \\
\text { ten Fälle } \\
\end{array}$} & \multicolumn{2}{|c|}{ Züchtung } & \multicolumn{2}{|c|}{ Agglutınation } \\
\hline & & $\begin{array}{c}\text { vorgenommen } \\
\text { in }\end{array}$ & $\underset{\text { in }}{\text { gelunget }}$ & $\begin{array}{c}\text { vorgenommen } \\
\text { in }\end{array}$ & $\begin{array}{c}\text { gelungen } \\
\text { in }\end{array}$ \\
\hline $\begin{array}{l}\text { leichte } \\
\text { mittelschwere } \\
\text { schwere }\end{array}$ & $\begin{array}{l}20 \\
69 \\
21\end{array}$ & $\begin{array}{l}18 \\
68 \\
19\end{array}$ & $\begin{aligned} 1 & =6 \% \\
46 & =68 \% \\
16 & =84 \%\end{aligned}$ & $\begin{array}{l}20 \\
69 \\
21\end{array}$ & $\begin{array}{l}20=100 \% \\
65=94 \% \\
16=76 \%\end{array}$ \\
\hline Summa & 110 & 105 & $63=60 \%$ & 110 & $101=92 \%$ \\
\hline
\end{tabular}

Die Verhältnisse haben sich in der zweiten Woche nach der Richtung hin entwickelt, die in der ersten bereits angedeutet war. Unter den leichten Fällen gelang eine Züchtıng kaum mehr, für die inittelschweren ist sie etwas seltener geworden, ist aber immer noch in weit mehr als der Hälfte der Fälle vorhanden, für die schwereren ist eill negativer Ausfall der Züchtungsversuche beinahe die Ausnahme.

Anders verhält sich die Agglutinationsreaktion: sie zeigt sich beinahe für alle Fälle konstant, wenigstens angedeutet (die schwächere Reaktion 1/50 makroskopisch oder 1/100 mikroskopisch sind eingerechnet). Immerhin unterscheiden sich aber die schweren von den leichten Fällen aucil darin, indem hier die Aussicht auf einen positiven Ausfall beinahe sicher, dort nur wahrscheinlich ist.

\section{Tabelle 111 .}

Uebersicht über die Ergebnisse von Blutzüchtung und Agglutination in der dritten $\mathrm{Krankheitswoche.}$

\begin{tabular}{|c|c|c|c|c|c|}
\hline $\begin{array}{l}\begin{array}{l}\text { Verlaufsart der } \\
\text { Fälle }\end{array} \\
\end{array}$ & $\begin{array}{l}\text { Zahl der } \\
\text { untersuch- } \\
\text { ten Fálle }\end{array}$ & $\begin{array}{c}\text { Zü } \\
\text { vorgeti.। } \\
\text { in }\end{array}$ & $\begin{array}{l}\text { htung } \\
\text { gelungen } \\
\text { in }\end{array}$ & $\begin{array}{c}\text { Agg } \\
\text { vorgen. } \\
\text { in }\end{array}$ & $\begin{array}{c}\text { utination } \\
\text { gelungen } \\
\text { in }\end{array}$ \\
\hline $\begin{array}{c}\text { leicht } \\
\text { mittelschwer } \\
\text { schwere } \\
\end{array}$ & $\begin{array}{r}6 \\
23 \\
15 \\
\end{array}$ & $\begin{array}{r}6 \\
23 \\
13 \\
\end{array}$ & $\begin{array}{l}\stackrel{0}{9} \% \\
9=40 \% \\
9=70 \%\end{array}$ & $\begin{array}{r}6 \\
23 \\
15 \\
\end{array}$ & $\begin{aligned} 6 & =100 \% \\
20 & =87 \% \\
13 & =90 \%\end{aligned}$ \\
\hline Summa & 44 & 42 & $18=43 \%$ & 44 & $39=91 \% / n$ \\
\hline
\end{tabular}

Tabelle IV.

Uebersicht über die Ergebnisse von Blutzüchtung und Agglutination in der vierten Krankheitswoche und spater.

\begin{tabular}{|c|c|c|c|c|c|}
\hline \multirow{2}{*}{$\begin{array}{c}\text { Verlaufart der } \\
\text { Fälle }\end{array}$} & \multirow{2}{*}{$\begin{array}{c}\text { Zahl der } \\
\text { untersuch. } \\
\text { ten Fäle } \\
\end{array}$} & \multicolumn{2}{|c|}{ Züchtung } & \multicolumn{2}{|c|}{ Agglutination } \\
\hline & & $\begin{array}{c}\text { vorgen. } \\
\text { in }\end{array}$ & $\underset{\text { in }}{\text { gelungen }}$ & $\begin{array}{c}\text { vorgen } \\
\text { in }\end{array}$ & $\begin{array}{c}\text { gelungen } \\
\text { in }\end{array}$ \\
\hline $\begin{array}{c}\text { leicht } \\
\text { mittelschwer } \\
\text { schwere }\end{array}$ & $\begin{array}{l}7 \\
9 \\
4 \\
\end{array}$ & $\begin{array}{l}7 \\
7 \\
4\end{array}$ & $\begin{array}{c}0 \\
0 \\
4=100^{o} \%\end{array}$ & $\begin{array}{l}6 \\
8 \\
4 \\
\end{array}$ & $\begin{array}{l}6=100 \% \\
6=75 \% \\
3=75 \%\end{array}$ \\
\hline Summa & 20 & 18 & $4=22 \%$ & 18 & $15=83 \%$ \\
\hline
\end{tabular}

Diese beiden Zusammenstellungen zeigen, dal von der dritten Woche ab der Züchtungsversuch als diagnostisches Hilfsmittel fortfällt. Die Aussicht auf einen positiven Ausfall ist gering. Werden Bazillen gefunden, so handelt es sich immer um schwerere bis sehr schwere Fälle. Die Prognose ist wohl stets ungünstig.

Die Agglutininbildung scheint beinahe in allen Fällen gleichmäbig leicht und konstant vor sich zu gehen. Für die Bedeutung dieser Verhältnisse beziehe ich mich auf das oben Gesagte. Aber es zeigt sich wieder aufs neue, dab die Vorgtinge der Heilung und die Bildung von Agglutininen nicht entfernt aneinander gebunden sind.

Paratyphus. Die Mehrzahl der 13 untersuchten Paratyphusfalle waren in Auftreten und Verlauf Krankheiten leichterer Natur. Doch beobachteten wir auch Formen schwerster und mittlerer Art.

Die schweren Paratyphen (zwei Fälle vom Typus B) verhielten sich bakterizidisch genau wie schwere Typhen, vielleicht mit dem Unterschied, daß die Agglutination dort frühzeitiger eintrat als hier. Die mittels chweren Fälle ließen die Züchtung meist vermissen. Freilich fehlen solche Fälle gerade für die erste Woche. In der zweiten Woche kommt auf drei Züchtungsversuche immerhin doch ein gelungener. Für die leichten Fälle verhält es sich ähnlich. Wir haben in der ersten Woche von etwa drei Fällen einmal eine gelungene Züchtung zu verzeichnen. Die Verhältnisse zahlenmäßig aus- zudrücken, hat bei der kleinen Menge der untersuchten Fälle keinen Sinn.

Bei unbefangener Beobachtung gewinnt man das Urteil, dab es sich beim Paratyphus gewöhnlich um leichtere Formen des Abdominaltyphus handelt, dab aber die Reaktion des Organismus auf das infizierende Agens sich sehr ähnlich, wenn nichtgenau wie bei der Infektion vom Typus Eberth verhält. Jedenfalls läßt sich ein irgendwie typisches Verhalten nicht feststellen.

Wir reden hier von dem rein klinischen Bild. Daf bezüglich einer Immunität, dem einwandfreiesten Kriterium für die Identifizierung zweier Mikroorganismen, sich die beiden Erreger, resp. die von ihnen hervorgerufenen Krankheiten, gänzlich unabhängig voneinander zeigen, soll hernach an der Hand eines Falles gezeigt werden.

Die Diagnose Paratyphus wurde nach den bakteriologischen Prinzipien gestellt. Von vornherein klar war sie bei den wenigen Fällen, bei denen aus dem Blute Paratyphusbazillen gezüchtet werden konnten. Es traf dies bei drei Fiillen zll, alle vom Typus B. Demnächst entscheidend war Buzillenzüchtung aus dem Stuhl, die in zwei Fällen gelang. In der Mehrzahl der Fälle muite die Agglutinationsreaktion das letzte Wort sprechen. Gerade diese diagnostische Stütze ist bekanntlich eine der schwerer deutbaren und von vornherein nicht absolut entscheidenden. Man richtete sich zur diagnostischen Beurteilung nach den gegenwärtig geltenden Anschauungen, wie sie von Castellani, Kay ser und de Feyfu, Jürgens u. a. festgelegt wurden. Tritt die Agglutination nur mit Paratyphusbazillen ein und verschwindet diese Agglutinationskraft nicht, nachdem das Serum Init Typhusbazillen abgesättigtwurde (Castellani u. a.), so wird Paratyphus angenommen, wenn die Reaktion im Laufe der Krankheit mehrere Male sich in der gleichen Weise wiederholt oder gar an Starke zunimmt. So wurde in fünf Fällen die Diagnose auf Paratyphus B, in einem auf Paratyphus A gestellt.

Ein Fall, bei dem die klinische Diagnose auf Abdominaltyphus gestellt wurde, agglutinierte schon am dritten Tage Typhusbazillen, $1: 50$, gab also eine schwache, nicht eigentlich beweisende Reaktion für Typhus. Fünf Tage später war nur für Paratyphusbazillen B in einer Verdünnung von $1 / 100$ Agglutination vorhanden.

In einem andern Falle von klinischem Abdominaltyphus, der zunächst leicht verlief, nach achttägigem Spitalaufenthalt - er kam am achten Tage der Erkrankung herein - entfiebert war, fand sich am elften Tage der Krankheit Agglutination für Typhus, Paratyphus A und B $1 / 100$. Der Castellanische Versuch unterblieb leider. 15 Tage nach der Entfieberung stieg am Abend des 16. die Temperatur auf 40,5 . Es begann ein fieberhafter Zustand, der klinisch ganz anders aussah als die erste Erkrankung, mit einer zwölftägigen Continua von etwa immer $40,5^{\circ}$ einherging und dann allmảhlich abermals zur Entfieberung kam. Am ersten Tage dieser neuerlichen Erkrankung wurden Paratyphusbazillen B 1/100 agglatiniert, vier Tage später stellte sich der Grenzwert der Agglutination für Paratyphusbazillen $1 / 200$ fest. Die Züchtung ergab immer negative Resultate. Funf Tage nach der zweiten Deferveszenz nochmaliger Anstieg der Temperatur von $36,5^{\bullet}$ morgens bis $40,5^{\bullet}$ mittags, sechstägige weitere sehr hohe Continuą; am 15. Tage dieses zweiten Rezidivs Entfieberung. Klinisch sahen sich die beiden letzten Erkrankungen durchaus ahnlich. Bakteriologisch ergab die Untersuchung im dritten Fieberstadium von Bazillen wieder nichts, hingegen eine Agglutination für Typhusbazillen 1/10e. Der Bakterizidieversuch, der am 20. Tage der Rekonvaleszenz angestellt wurde, ergab, daß $0,01 \mathrm{~g}$ Patientenserum ein Meerschweinchen von $300 \mathrm{~g}$ vor der vierfachen letalen Dosis Typhusbazillen schutzte.

Ueber die Deutung des Falles, der klinisch in seinem zweiten Krankheitsstadium nicht als eigentliches Rezidiv, sondern als wirkliche Neuerkrankung angesehen wurde, eine Neuerkrankung, der dann erst ein echtes Rezidiv folgte, ist von bakteriologischer Seite das ganz entsprechende Urteil abgegeben, daß es sich im ersten Stadium um einen Paratyphus B mit initialer Gruppenagglutination (s. o.) gehandelt haben kann, dessen spezifische Serumreaktion bis in die ersten Tage der zweiten Erkrankung (an Typhus), hineindauerte. Der Typhus gab sich bakteriologisch erst im Rezidiv durch die sofortige Agglutination für Typhusbazillen, sowie den positiven Pfeifferschen Versuch (s. o.) zu erkennen. Es wïrde sich also um Typhus nach Paratyphus handeln, eine Erschei- 
nung, auf deren eminent praktische Bedeutung neuerdings wieder von Levy und Gaethgens ${ }^{1}$ ) aufmerksam gemacht wurde. 